\title{
Evaluation of the Pharmacokinetic Interaction and Safety of Atogepant Co-Administered with Acetaminophen or Naproxen in Healthy Participants: A Randomized Trial
}

\author{
Ramesh Boinpally ${ }^{1} \cdot$ John Spaventa ${ }^{1} \cdot K_{\text {Kala Chen }}^{2} \cdot$ Matthew Butler $^{3}$
}

Accepted: 30 March 2021 / Published online: 5 May 2021

(c) The Author(s) 2021

\begin{abstract}
Background Atogepant is an oral calcitonin gene-related peptide (CGRP) receptor antagonist in development for preventive treatment of migraine.

Objective To evaluate potential pharmacokinetic drug-drug interactions (DDIs), safety and tolerability of atogepant coadministered with acetaminophen or naproxen in healthy participants.

Methods This open-label, randomized, five-way crossover, single-center, phase 1 DDI trial randomized healthy adult participants to one of ten intervention sequences to receive single-dose $60 \mathrm{mg}$ atogepant, $1000 \mathrm{mg}$ acetaminophen, $500 \mathrm{mg}$ naproxen, or co-administrations of atogepant with acetaminophen or naproxen, with 7-day washout periods between interventions. Potential DDIs were assessed using geometric mean ratios and $90 \%$ confidence intervals (CIs) calculated from maximum plasma drug concentrations $\left(C_{\max }\right)$ and area under the plasma drug concentration-time curves (AUCs) for co-administered medications versus medications administered alone. Secondary pharmacokinetic parameters [time to $C_{\max }\left(t_{\max }\right)$, terminal elimination half-life $\left(t_{1 / 2}\right)$, volume of distribution during terminal phase $\left(V_{\mathrm{Z}} / F\right)$, total body clearance $\left.(\mathrm{CL} / F)\right]$, and safety were evaluated.

Results Forty participants enrolled; 35 (87.5\%) completed the trial. Atogepant $C_{\max }$ was unchanged, AUC $\mathrm{A}_{0-t}$ and $\mathrm{AUC}_{0-\infty}$ both increased $13 \%$, and $t_{\max }$ and $t_{1 / 2}$ were unchanged when co-administered with acetaminophen; and acetaminophen $C_{\max }$ decreased $11 \%, \mathrm{AUC}_{0-t}$ and $\mathrm{AUC}_{0-\infty}$ both decreased $6 \%$, and $t_{\max }$ and $t_{1 / 2}$ were unchanged when co-administered with atogepant. Atogepant mean (SD) $V_{\mathrm{z}} / F$ and CL/F were $369.45(255.68) \mathrm{L}$ and $18.88(9.28) \mathrm{L} / \mathrm{h}$, respectively, when administered alone and $297.56(196.01) \mathrm{L}$ and $16.33(6.11) \mathrm{L} / \mathrm{h}$ when co-administered with acetaminophen. Atogepant $C_{\max }$ was unchanged, $\mathrm{AUC}_{0-t}$ and $\mathrm{AUC}_{0-\infty}$ both decreased $1 \%$, and $t_{\max }$ and $t_{1 / 2}$ were unchanged when co-administered with naproxen; and naproxen $C_{\max }$ decreased $6 \%, \mathrm{AUC}_{0-t}$ and $\mathrm{AUC}_{0-\infty}$ both decreased $2 \%$, and $t_{\max }$ and $t_{1 / 2}$ were unchanged when co-administered with atogepant. Atogepant mean (SD) $V_{z} / F$ and CL/F were 359.61 (247.99) L and 18.80 (7.78) L/h, respectively, when co-administered with naproxen. Treatment-emergent adverse events (TEAEs) occurred at rates of 5.6-21.1\% across interventions. The most commonly reported TEAEs were oropharyngeal pain $(n=2$, with atogepant; not treatment related) and nausea ( $n=2$, with atogepant/acetaminophen; treatment related).

Conclusion Co-administration of $60 \mathrm{mg}$ atogepant with $1000 \mathrm{mg}$ acetaminophen or $500 \mathrm{mg}$ naproxen was safe and well tolerated in healthy participants, and no DDIs were observed.
\end{abstract}

\section{Introduction}

Ramesh Boinpally

Ramesh.Boinpally@AbbVie.com

1 Clinical Pharmacology, AbbVie, 5 Giralda Farms, Madison, NJ 07940, USA

2 Data \& Statistical Sciences, AbbVie, Madison, NJ, USA

3 Global Patient Safety \& Epidemiology, AbbVie, Madison, NJ, USA
Migraine is a complex, chronic disease with recurrent attacks that are often incapacitating and characterized by headache pain as well as neurologic and autonomic symptoms [1-5]. Migraine is highly prevalent, affecting more than 1 billion individuals worldwide [6], and is a leading cause of years lived with disability in those under 50 years of age, resulting in high global and individual burden of disease [7, 8]. Migraine management consists of two types of medications: acute medications, used for the treatment of 


\section{Key Points}

Atogepant, an oral calcitonin gene-related peptide (CGRP) receptor antagonist that is in development for the preventive treatment of migraine, is likely to be co-administered with the analgesic medications acetaminophen or naproxen.

In this phase 1, open-label, drug-drug interaction study, atogepant overall exposure (area under the plasma drug concentration-time curve) was similar when administered alone or co-administered with acetaminophen or naproxen; peak atogepant plasma concentration, time to peak plasma concentration, and atogepant half-life were also similar when co-administered with acetaminophen or naproxen.

Co-administration of atogepant with acetaminophen or naproxen appeared safe and no clinically significant drug-drug interactions were observed.

a migraine attack and associated symptoms, and preventive medications, used to reduce attack frequency, severity, duration, and disability $[9,10]$. Commonly used over-the-counter acute treatments for mild to moderate migraine attacks include nonsteroidal anti-inflammatory drugs (NSAIDs), nonopioid analgesics, and acetaminophen [9, 11-13]. Acetaminophen and naproxen are often used in combination with other medications $[14,15]$. They are modestly effective for the acute treatment of migraine attacks that are mild to moderate in severity, and are generally well tolerated [14]. Triptans, dihydroergotamine, and gepants are migraine-specific medications recommended for the acute treatment of moderate to severe migraine attacks $[9,16,17]$.

Preventive treatment is considered when migraine attacks are frequent (generally around four or more monthly headache days); attacks interfere with daily activities despite acute treatment; contraindication to, failure, or overuse of acute treatments exists; if the acute treatments are associated with adverse events (AEs); or the patient prefers preventive treatment over acute treatment [9]. Preventive treatments include migraine-nonspecific medications (beta blockers, antiepileptics, calcium channel blockers, antidepressants, and onabotulinumtoxin A) $[9,10]$. Recently, new pharmacotherapies that target the calcitonin gene-related peptide (CGRP) or its receptors have demonstrated efficacy and been approved for the acute treatment of migraine attacks (ubrogepant [16], rimegepant [17]) and for the preventive treatment of migraine in adults (fremanezumab
[18], galcanezumab [19], erenumab [20], eptinezumab [21]) [22].

Atogepant is an oral CGRP receptor antagonist currently in development for the preventive treatment of migraine. In a phase $2 \mathrm{~b} / 3$, randomized, controlled trial (NCT02848326), treatment with atogepant led to significant reductions in mean monthly migraine days and was well tolerated in adults with a history of migraine (4-14 migraine days per month) [23]. A subsequent 12-week, randomized, placebocontrolled, multidose phase 3 clinical trial (NCT03777059) of the use of atogepant for migraine prevention reported a favorable safety profile with efficacy at all doses of atogepant compared with placebo [24]. When used for migraine prevention, atogepant is likely to be co-administered with acute medications for the treatment of breakthrough migraine attacks, including acetaminophen and naproxen. Here we report the results from a phase 1 trial conducted to assess the potential for pharmacokinetic drug-drug interactions between atogepant and acetaminophen and between atogepant and naproxen in healthy adult participants.

\section{Methods}

\subsection{Study Design}

This open-label, randomized, five-way crossover, phase 1 trial assessed the potential pharmacokinetic interaction between atogepant and acetaminophen, and atogepant and naproxen in healthy adult participants. The trial was conducted at a single site in the USA from 20 April 2019 through 24 June 2019. Eligible participants were randomly assigned to one of ten intervention sequences to receive a single oral dose of $60 \mathrm{mg}$ atogepant $(1 \times 60 \mathrm{mg}$ tablet; Allergan plc, Dublin, Ireland), a single dose of $1000 \mathrm{mg}$ acetaminophen $(2 \times 500 \mathrm{mg}$ caplet; Tylenol Extra Strength caplet; McNeil Consumer Healthcare, Fort Washington, PA, USA), co-administration of $60 \mathrm{mg}$ atogepant and $1000 \mathrm{mg}$ acetaminophen, a single dose of $500 \mathrm{mg}$ naproxen $(1 \times 550$ $\mathrm{mg}$ naproxen sodium tablet equivalent to $500 \mathrm{mg}$ naproxen; Anaprox DS tablet; Canton Laboratories, Alpharetta, GA, USA), and co-administration of $60 \mathrm{mg}$ atogepant and 500 $\mathrm{mg}$ naproxen under fasted conditions $(10 \mathrm{~h}$ prior to dosing and $4 \mathrm{~h}$ following dose administration) after a screening period of up to 21 days. Participants were randomized based on a schedule prepared by the study sponsor according to a $5 \times 5$ Williams Squares design (Supplemental Table S1, Online Supplemental Material), and received interventions on days $1,8,15,22$, and 29 with a 7 -day washout between interventions. A follow-up period of 30 days occurred after the last dose of study intervention on day 29. Because this was an open-label trial, investigators and participants were not blinded to interventions. 
The study protocol was approved by Bio-Kinetic Clinical Applications Institutional Review Board (Springfield, MO, USA). All participants provided written informed consent prior to initiation of any study-specific procedures. This study was conducted in accordance with the principles of the Declaration of Helsinki and the International Council for Harmonisation (ICH) E6 guideline for Good Clinical Practice.

\subsection{Participants}

Eligible participants were healthy adults, 18-45 years of age (inclusive), nonsmokers/nonusers of nicotine-containing products (never used or had not used within the previous 2 years), with a body mass index (BMI) $\geq 18$ and $\leq 30 \mathrm{~kg}$ / $\mathrm{m}^{2}$ and sitting pulse rate $\geq 45$ and $\leq 100$ beats per minute (bpm). Participants with childbearing potential had to use contraception during the study, and females had to have a negative pregnancy result on day -1 and could not be breastfeeding.

Exclusion criteria included sitting systolic blood pressure (BP) $\geq 140 \mathrm{mmHg}$ or $\leq 90 \mathrm{mmHg}$; sitting diastolic $\mathrm{BP} \geq 90$ $\mathrm{mmHg}$ or $\leq 50 \mathrm{mmHg}$; abnormal electrocardiogram (ECG) results thought to be potentially clinically significant; or QT prolongation (QTcF $\geq 450 \mathrm{~ms}$ or $\geq 470 \mathrm{~ms}$ for males or females, respectively). Participants could not test positive for benzoylecgonine, methadone, barbiturates, amphetamines, benzodiazepines, alcohol, cannabinoids, opioids, phencyclidine, or cotinine; have a clinically significant disease state; have a clinical condition that might affect the absorption, distribution, biotransformation, or excretion of atogepant, acetaminophen, or naproxen; have a history of alcohol or other substance abuse within the previous 5 years; have previously participated in an investigational study of atogepant; or have participated in a clinical investigation (within 30 days before first administration), blood donation program (within 60 days before first administration) or plasma donation program (within 30 days before first administration).

Participants could not have consumed beverages, food, herbs, or dietary supplements that could affect various drugmetabolizing enzymes and transporters within 14 days prior to dosing and through the end-of-dosing visit. Participants also had to abstain from drinking alcohol $72 \mathrm{~h}$ before dosing through follow-up and abstain from ingesting caffeine- or xanthine-containing products for $48 \mathrm{~h}$ before dosing through the collection of the last blood sample for pharmacokinetic testing.

\subsection{Study Procedures}

Participants were admitted to the study center on days -1 , $7,14,21$, and 28 where they remained for $48 \mathrm{~h}$ after each intervention with the exception of administration of acetaminophen alone, when participants remained for $24 \mathrm{~h}$ after intervention. Study interventions were administered under fasting conditions (10-h overnight fast) with approximately $240 \mathrm{~mL}$ of water on days $1,8,15,22$, and 29. Participants remained seated and upright for $4 \mathrm{~h}$ after dosing. After all interventions had been administered, participants returned to the study center within 7 days of day 32 (day 30 for participants who received acetaminophen alone as their final intervention) and at day $59( \pm 3)$ for safety evaluations.

\subsection{Pharmacokinetic Assessments}

Blood samples were collected into prechilled 4-mL collection tubes (Vacutainer, BD, Franklin Lakes, NJ, USA) containing $\mathrm{K}_{2}$ EDTA as an anticoagulant for atogepant or naproxen pharmacokinetic testing at 0 (predose), 0.5, 1 , 1.5, 2, 3, 4, 6, 8, 12, 16, 24, 36, 48, and $72 \mathrm{~h}$ after dosing; samples for acetaminophen pharmacokinetic testing were collected at the same time points through $24 \mathrm{~h}$. Atogepant, acetaminophen, and naproxen concentrations in plasma samples were measured using separate validated liquid chromatography with tandem mass spectrometry assays. The lower limit of quantitation in plasma was $1.0 \mathrm{ng} / \mathrm{mL}$ for atogepant, $0.1 \mu \mathrm{g} / \mathrm{mL}$ for acetaminophen, and $0.5 \mu \mathrm{g} /$ $\mathrm{mL}$ for naproxen.

\subsection{Bioanalytical Assay}

Atogepant and naproxen were extracted from $0.100 \mathrm{~mL}$ and $0.0500 \mathrm{~mL}$ of human plasma, respectively, by protein precipitation extraction methods. Acetaminophen was extracted from $0.100 \mathrm{~mL}$ of human plasma by liquid-liquid extraction methods. Internal standards for atogepant, naproxen, and acetaminophen were MK-8031-D3, naproxen-D3, and acetaminophen-D4, respectively. The analytes were identified and quantified using reversed-phase high-performance liquid chromatography with triple quadrupole mass spectrometry detection over a theoretical concentration range of $1.00 \mathrm{ng} / \mathrm{mL}$ to $1000.00 \mathrm{ng} / \mathrm{mL}$ of atogepant, $0.50 \mu \mathrm{g} / \mathrm{mL}$ to $100.00 \mu \mathrm{g} / \mathrm{mL}$ of naproxen, and $0.10 \mu \mathrm{g} / \mathrm{mL}$ to $30.00 \mu \mathrm{g} /$ $\mathrm{mL}$ of acetaminophen. The identity of the reference standard (MK-8031-D3) had to be met and identified. The matrix used to prepare calibrants and quality control samples was screened for potential interference at the retention times and mass transitions of atogepant and MK-8031-D3, naproxen and naproxen-D3, and acetaminophen and acetaminophenD4. In addition to blank and zero calibrants, 11 non-zero calibrants (ten for naproxen and acetaminophen) and three levels of quality control samples (four for acetaminophen) containing atogepant were prepared with analyte-free human plasma, using $\mathrm{K}_{2}$ EDTA as anticoagulant. When the analyte was atogepant, calibrant concentrations ranged from $1.00 \mathrm{ng} /$ 
$\mathrm{mL}$ to $1000.00 \mathrm{ng} / \mathrm{mL}$, and quality control sample concentrations were $3.00 \mathrm{ng} / \mathrm{mL}, 500.00 \mathrm{ng} / \mathrm{mL}$, and $750.00 \mathrm{ng} / \mathrm{mL}$. When the analyte was naproxen, calibrant concentrations ranged from $0.50 \mu \mathrm{g} / \mathrm{mL}$ to $100.00 \mu \mathrm{g} / \mathrm{mL}$ and quality control sample concentrations were $1.50 \mu \mathrm{g} / \mathrm{mL}, 20.00 \mu \mathrm{g} / \mathrm{mL}$, and $75.00 \mu \mathrm{g} / \mathrm{mL}$. When the analyte was acetaminophen, calibrant concentrations ranged from $0.10 \mu \mathrm{g} / \mathrm{mL}$ to 30.00 $\mu \mathrm{g} / \mathrm{mL}$ and quality control sample concentrations were 0.30 $\mu \mathrm{g} / \mathrm{mL}, 5.00 \mu \mathrm{g} / \mathrm{mL}, 15.00 \mu \mathrm{g} / \mathrm{mL}$, and $22.50 \mu \mathrm{g} / \mathrm{mL}$.

\subsection{Study Endpoints}

Pharmacokinetic parameters of area under the plasma drug concentration-time curve from time 0 to time $\mathrm{t}\left(\mathrm{AUC}_{0-t}\right)$ and to infinity $\left(\mathrm{AUC}_{0-\infty}\right)$, and maximum plasma concentration $\left(C_{\max }\right)$ were used to evaluate interactions between atogepant and acetaminophen, and between atogepant and naproxen administered to healthy adults. Additional pharmacokinetic parameters [time to $C_{\max }\left(t_{\max }\right)$, apparent terminal elimination half-life $\left(t_{1 / 2}\right)$, apparent total body clearance from plasma after extravascular administration $(\mathrm{CL} / F)$, and apparent volume of distribution during the terminal phase after extravascular administration $\left.\left(V_{\mathrm{Z}} / F\right)\right]$ for atogepant, acetaminophen, and naproxen, and safety and tolerability were evaluated.

\subsection{Safety}

Safety endpoints included the incidence and types of treatment-emergent adverse events (TEAEs), serious adverse events (SAEs), physical examinations, clinical laboratory tests, vital signs, and ECGs.

\subsection{Statistical Analyses}

A sample size of 40 participants with at least 28 completing the study was estimated to provide at least $90 \%$ power to show that the $90 \%$ confidence intervals (CIs) for the ratio of geometric mean values for $C_{\max }$ and AUC of atogepant with and without co-administration of acetaminophen or naproxen were within $80-125 \%$. This sample size was based on the following assumptions: the within-participant coefficient of variation is $25 \%$ for atogepant $C_{\max }$ and AUC; the true geometric mean ratios (GMRs) of test:reference were approximately 1 ; and the naproxen within-participant coefficient of variation after oral administration is lower at $18 \%$ for $C_{\max }$ and $20 \%$ for AUC. This sample size also assumed a large dropout rate based on the length of the intervention periods and the number of interventions.

Pharmacokinetic parameters derived from plasma concentrations included $C_{\text {max }}, \mathrm{AUC}_{0-t}, \mathrm{AUC}_{0-\infty}, t_{\max }, t_{1 / 2}, \mathrm{CL} / F$, and $V_{Z} / F$ and were calculated from plasma concentrations using Phoenix WinNonlin version 8.0 software. A linear mixed-effects model was used for the comparison of atogepant, acetaminophen, and naproxen log-transformed pharmacokinetic parameters $\left(C_{\max }, \mathrm{AUC}_{0-t}, \mathrm{AUC}_{0-\infty}\right)$ to co-administrations of atogepant with acetaminophen, and atogepant with naproxen. In this model, intervention, period, and sequence were fixed effects and participant within sequence was a random effect. To evaluate drug-drug interactions, two-sided 90\% CIs for the GMRs between the test intervention (co-administration of atogepant with acetaminophen or naproxen) and reference intervention (atogepant, acetaminophen, or naproxen alone) were constructed. In accordance with US Food and Drug Administration (FDA) guidance for studies of drug-drug interactions [25], no significant effect between the test and reference interventions was concluded if the $90 \%$ CIs for the GMRs were within $80-125 \%$.

A TEAE was defined as any untoward medical occurrence in a study participant, temporally associated with the use of study intervention, whether or not considered related to the study intervention. The TEAE could include any unfavorable and unintended sign (including an abnormal laboratory finding), symptoms, or disease (new or exacerbated) temporally associated with the use of study intervention. An SAE was defined as any untoward medical occurrence that at any dose resulted in death, was life threatening, required inpatient hospitalization or prolongation of an existing hospital stay, resulted in persistent disability or incapacity, was a congenital anomaly or birth defect, or resulted from some other situation that may include: invasive or malignant cancers, intensive intervention in an emergency room or at home for allergic bronchospasm, blood dyscrasias or convulsions that do not result in hospitalization, or development of drug dependency or drug abuse. AE intensity was assessed as mild (AE that is usually transient and may require only minimal treatment or therapeutic intervention and does not generally interfere with usual activities of daily living), moderate (AE that is usually alleviated with additional specific therapeutic intervention and interferes with usual activities of daily living, causing discomfort but poses no significant or permanent risk of harm to the participant), and severe (AE that interrupts usual activities of daily living, or significantly affects clinical status, or may require intensive therapeutic intervention).

Safety analyses were conducted on the safety population, which comprised all participants who received at least one intervention dose. Pharmacokinetic analysis populations for atogepant with or without acetaminophen, atogepant with or without naproxen, acetaminophen with or without atogepant, and naproxen with or without atogepant included all participants who received the interventions 
and had evaluable pharmacokinetic parameters available for analysis.

\section{Results}

\subsection{Participants}

A total of 40 participants enrolled and were randomized; $35(87.5 \%)$ completed the study (Fig. 1). Five participants discontinued the study: three participants withdrew consent, and two were lost to follow-up. The mean age of the safety population was 30.0 years, approximately half were female $(47.5 \%)$, and most were White $(85.0 \%)$ (Table 1$)$. No notable differences in demographic characteristics between intervention groups were noted. The safety population comprised all 40 participants; populations analyzed for pharmacokinetic outcomes included 37 participants for atogepant with or without acetaminophen, 38 for acetaminophen with or without atogepant, 38 for atogepant with or without naproxen, and 36 for naproxen with or without atogepant.

\subsection{Atogepant-Acetaminophen Drug-Drug Interaction}

The mean (standard deviation; SD) plasma concentrations of atogepant with or without co-administered acetaminophen are shown in Fig. 2. The $C_{\max }$ and overall exposure (AUC) of atogepant were unchanged when atogepant was co-administered with acetaminophen (Table 2, Fig. 2). The median atogepant $t_{\max }$ and mean apparent terminal $t_{1 / 2}$ of atogepant with or without co-administration of acetaminophen were generally similar.

The mean (SD) plasma concentrations of acetaminophen administered alone or co-administered with atogepant are shown in Fig. 3a. There were no clinically relevant changes in acetaminophen pharmacokinetic parameters when coadministered with atogepant compared with acetaminophen administered alone (Table 3, Fig. 3a).

The GMRs and their 90\% CIs for the comparison of $C_{\text {max }}$ and AUC parameters of atogepant and acetaminophen co-administered versus administered alone are summarized in Table 4. Although atogepant $\mathrm{AUC}_{0-t}$ and
Fig. 1 Participant disposition. ${ }^{\text {aPharmacokinetic analysis }}$ populations included only those participants who had evaluable parameters

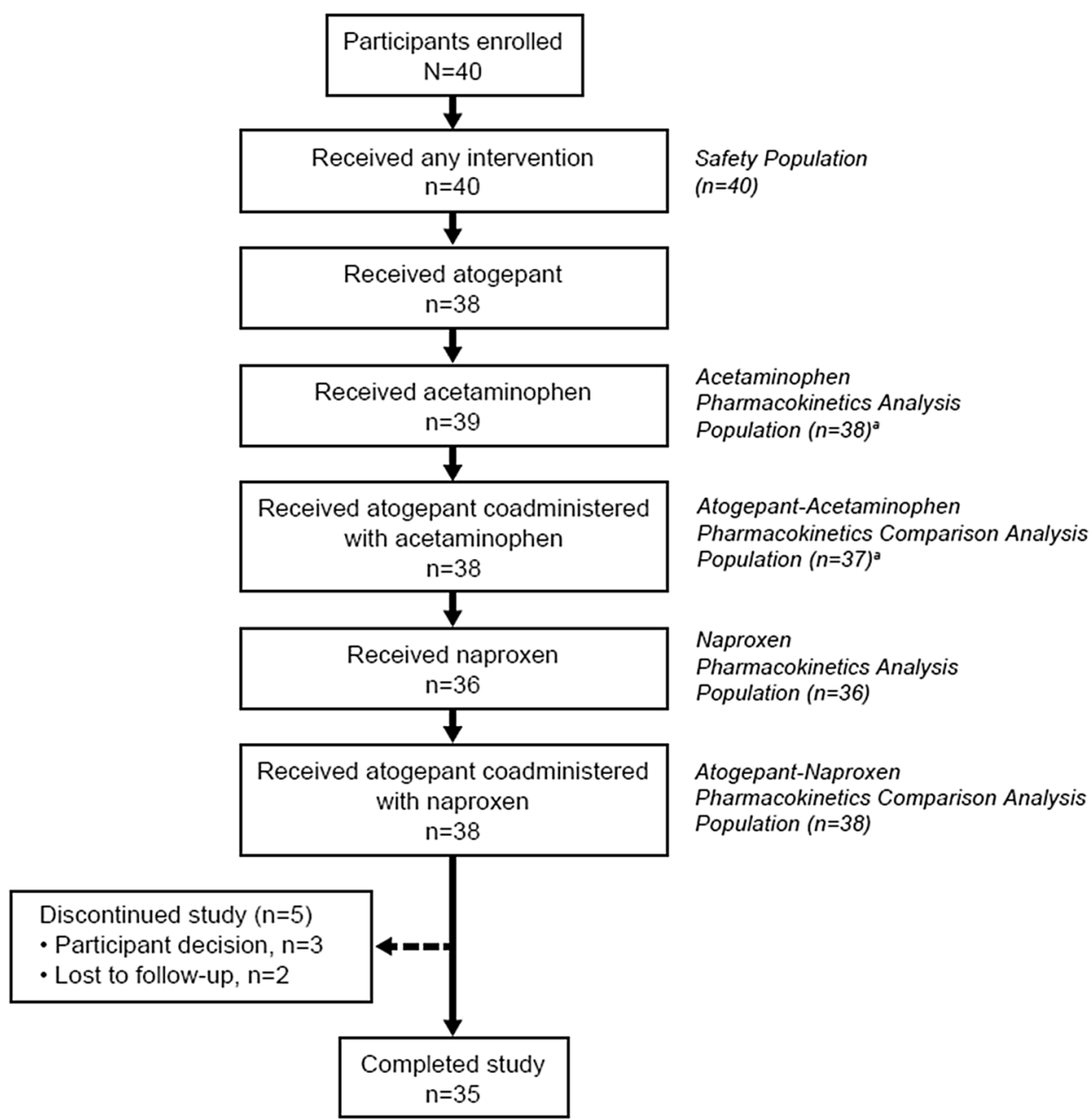


Table 1 Demographic characteristics at baseline

\begin{tabular}{|c|c|c|c|c|c|}
\hline Characteristic & $\begin{array}{l}\text { Atogepant } \\
60 \mathrm{mg} \\
(n=38)\end{array}$ & $\begin{array}{l}\text { Acetaminophen } \\
1000 \mathrm{mg} \\
(n=39)\end{array}$ & $\begin{array}{l}\text { Atogepant } 60 \mathrm{mg}+ \\
\text { acetaminophen } 1000 \mathrm{mg} \\
(n=38)\end{array}$ & $\begin{array}{l}\text { Naproxen } \\
500 \mathrm{mg} \\
(n=36)\end{array}$ & $\begin{array}{l}\text { Atogepant } 60 \mathrm{mg}+ \\
\text { naproxen } 500 \mathrm{mg} \\
(n=38)\end{array}$ \\
\hline Age, y & $29.7(8.2)$ & $30.0(8.4)$ & $30.1(8.4)$ & 29.7 (8.4) & $29.7(8.2)$ \\
\hline Male sex, $n(\%)$ & $19(50.0)$ & $20(51.3)$ & $19(50.0)$ & $17(47.2)$ & $19(50.0)$ \\
\hline \multicolumn{6}{|l|}{ Race, $n(\%)$} \\
\hline White & $34(89.5)$ & $34(87.2)$ & $32(84.2)$ & $32(88.9)$ & $34(89.5)$ \\
\hline Black/African American & $3(7.9)$ & $4(10.3)$ & $5(13.2)$ & $3(8.3)$ & $3(7.9)$ \\
\hline Asian & $1(2.6)$ & $1(2.6)$ & $1(2.6)$ & $1(2.8)$ & $1(2.6)$ \\
\hline Hispanic ethnicity, $n(\%)$ & $1(2.6)$ & $1(2.6)$ & $1(2.6)$ & $1(2.8)$ & $1(2.6)$ \\
\hline Weight, kg & $75.7(11.0)$ & $76.0(10.9)$ & $76.2(11.3)$ & $75.4(11.1)$ & $75.7(11.0)$ \\
\hline Height, cm & $169.2(9.7)$ & $169.3(9.6)$ & $169.3(9.8)$ & $169.0(9.9)$ & $169.2(9.7)$ \\
\hline BMI, $\mathrm{kg} / \mathrm{m}^{2}$ & $26.4(2.6)$ & $26.4(2.6)$ & $26.5(2.6)$ & $26.3(2.6)$ & $26.4(2.6)$ \\
\hline
\end{tabular}

Values are means (SD) unless otherwise indicated

$B M I$ body mass index, $S D$ standard deviation

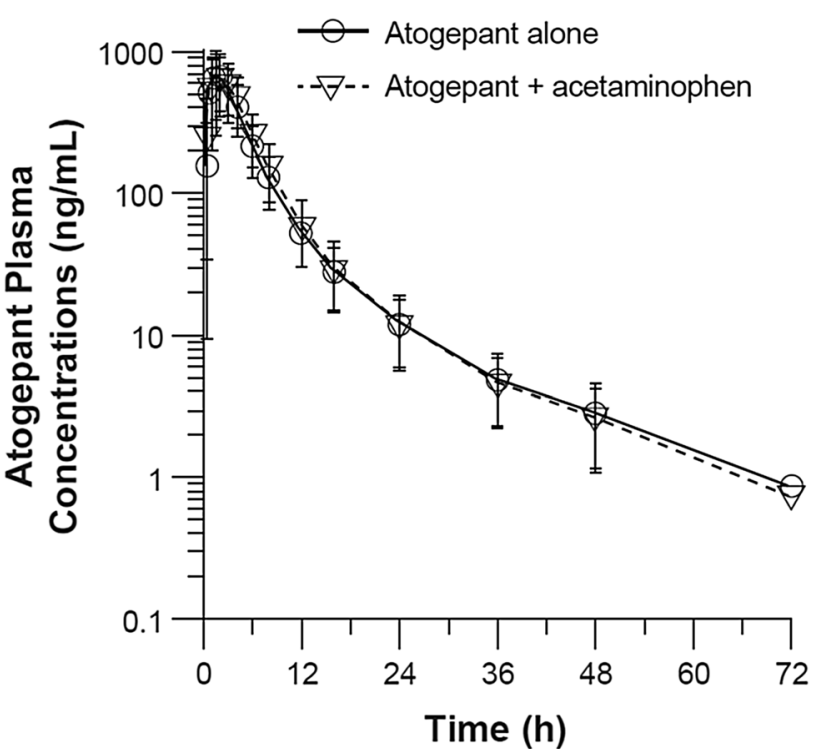

Fig. 2 Mean (SD) plasma atogepant concentration-time profile following single-dose oral administration of $60 \mathrm{mg}$ atogepant alone or when co-administered with $1000 \mathrm{mg}$ acetaminophen to fasted healthy participants on a semi-logarithmic scale

$\mathrm{AUC}_{0-\infty}$ were $13 \%$ greater when co-administered with acetaminophen, the $90 \%$ CIs were contained within the range of 0.80 and 1.25 for $\mathrm{AUC}_{0-t}, \mathrm{AUC}_{0-\infty}$, and $C_{\max }$, suggesting no drug-drug interaction. Similarly, while the $C_{\max }$ of a single dose of acetaminophen co-administered with atogepant decreased by $11 \%$ and AUCs decreased by $6 \%$, the $90 \%$ CIs were also contained within the range of 0.80 and 1.25 , suggesting no drug-drug interaction (Table 4).

\subsection{Atogepant-Naproxen Drug-Drug Interaction}

The mean (SD) plasma concentrations of atogepant with or without co-administered naproxen are shown in Fig. 4. The $C_{\max }$ and overall exposure (AUC) (Table 2, Fig. 4) of atogepant were unchanged when atogepant was co-administered with naproxen. The median atogepant $t_{\max }$ and mean apparent terminal $t_{1 / 2}$ of atogepant with or without co-administration of naproxen were generally similar.

The mean (SD) plasma concentrations of naproxen administered alone or co-administered with atogepant are shown in Fig. 3b. There were no clinically relevant changes in naproxen pharmacokinetic parameters when co-administered with atogepant compared with naproxen administered alone (Table 3, Fig. 3b).

The GMRs and their 90\% CIs for the comparison of $C_{\max }$ and AUC parameters of atogepant and naproxen co-administered versus administered alone are summarized in Table 5. Based on statistical comparisons using a linear fixedeffects model, the GMRs for atogepant $C_{\max }, \mathrm{AUC}_{0-t}$, and $\mathrm{AUC}_{0-\infty}$ when co-administered with naproxen were 1.00, 0.99 , and 0.99 , respectively. There was no drug-drug interaction effect of naproxen on the pharmacokinetics of atogepant because the $90 \%$ CIs were contained within the range of 0.80 and 1.25 for $\mathrm{AUC}_{0-t}, \mathrm{AUC}_{0-\infty}$, and $C_{\max }$ for atogepant when co-administered with naproxen versus administration alone. The GMRs for naproxen $C_{\max }, \mathrm{AUC}_{0-t}$, and $\mathrm{AUC}_{0-\infty}$ when co-administered with atogepant were $0.94,0.98$, and 0.98 , respectively. These findings, along with the fact that the $90 \%$ CIs were contained within the range of 0.80 and 1.25 for $C_{\max }$ and AUC, indicate no drug-drug interaction for naproxen when co-administered with atogepant versus administration alone. 
Table 2 Mean (SD) pharmacokinetic parameters of atogepant following single-dose oral administration of atogepant alone or when co-administered with acetaminophen or naproxen

\begin{tabular}{llll}
\hline Pharmacokinetic parameters & $\begin{array}{l}\text { Atogepant } 60 \mathrm{mg} \\
(n=38)\end{array}$ & $\begin{array}{l}\text { Atogepant 60 mg }+ \\
\text { acetaminophen 1000 mg } \\
(n=37)\end{array}$ & $\begin{array}{l}\text { Atogepant 60 mg }+ \\
\text { naproxen 500 mg } \\
(n=38)\end{array}$ \\
\hline$C_{\text {max }}(\mathrm{ng} / \mathrm{mL})$ & $788.10(327.82)$ & $761.56(339.08)$ & $765.26(257.10)$ \\
$\mathrm{AUC}_{0-t}(\mathrm{ng} \cdot \mathrm{h} / \mathrm{mL})$ & $3628.02(1201.26)$ & $4125.64(1428.11)$ & $3537.08(1081.78)$ \\
$\mathrm{AUC}_{0-\infty}(\mathrm{ng} \cdot \mathrm{h} / \mathrm{mL})$ & $3673.44(1208.61)$ & $4161.84(1434.23)$ & $3577.09(1088.98)$ \\
$t_{\max }(\mathrm{h})^{\mathrm{a}}$ & $1.50(1.00-4.00)$ & $2.00(1.00-4.00)$ & $2.00(1.00-3.00)$ \\
$t_{1 / 2}(\mathrm{~h})$ & $14.44(7.91)$ & $13.13(7.10)$ & $13.47(7.69)$ \\
$V_{\mathrm{z}} / F(\mathrm{~L})$ & $369.45(255.68)$ & $297.56(196.01)$ & $359.61(247.99)$ \\
$\mathrm{CL} / F(\mathrm{~L} / \mathrm{h})$ & $18.88(9.28)$ & $16.33(6.11)$ & $18.80(7.78)$ \\
\hline
\end{tabular}

$A U C_{0-t}$ area under the plasma drug concentration-time curve (AUC) from time 0 to time $t, A U C_{0-\infty}$ AUC from time 0 to infinity, $C L / F$ apparent total body clearance of drug from plasma after extravascular administration, $C_{\text {max }}$ maximum plasma drug concentration, $t_{1 / 2}$ terminal elimination half-life, $t_{\max }$ time to $C_{\max }, V_{z} / F$ apparent volume of distribution during the terminal phase after extravascular administration

${ }^{\mathrm{a}}$ Median (range)

a

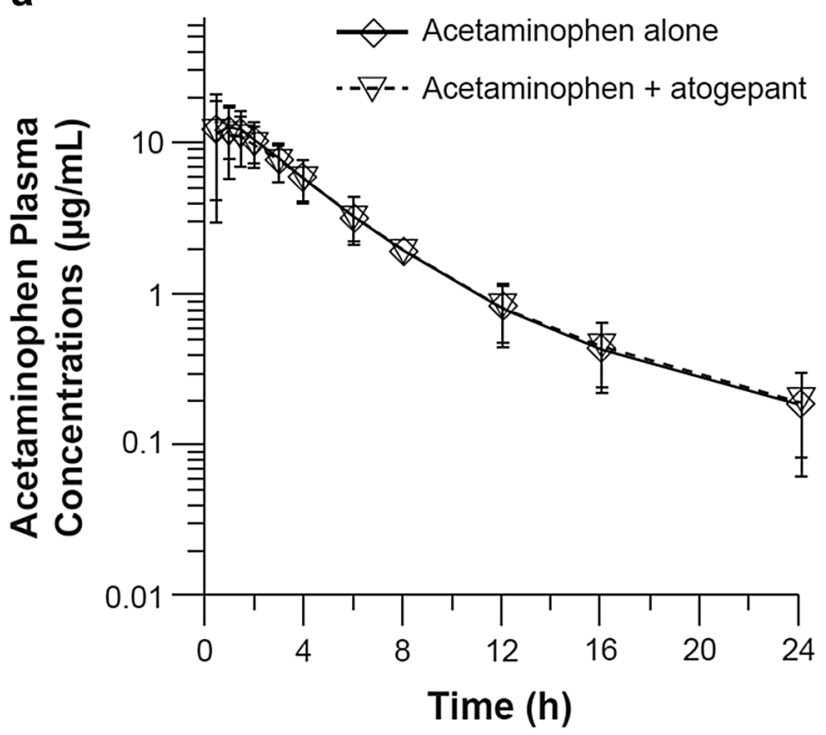

b

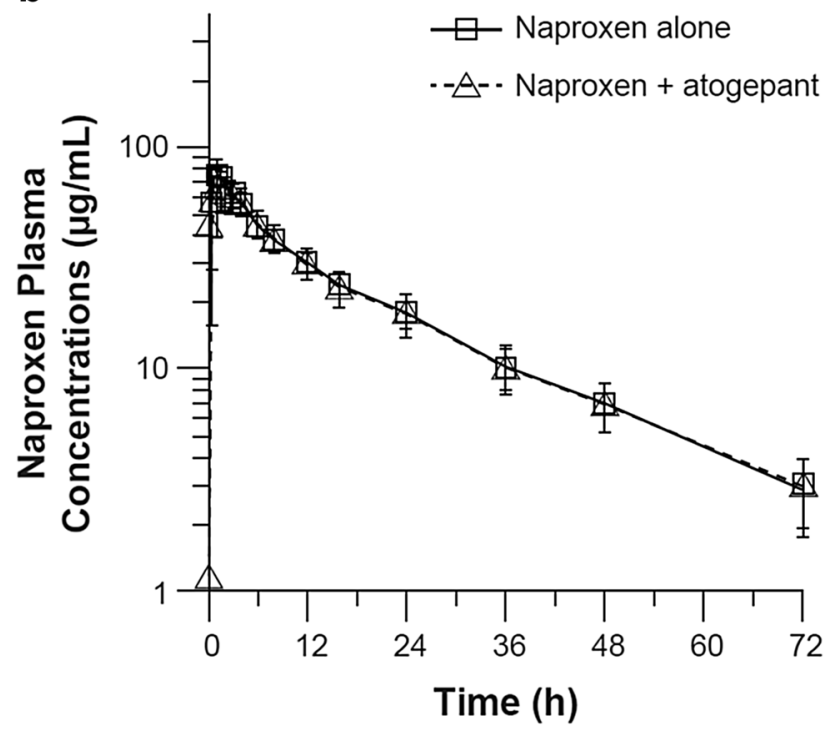

Fig. 3 Mean (SD) plasma acetaminophen (a) or naproxen (b) concentration-time profile following single-dose oral administration of 1000 mg acetaminophen or $500 \mathrm{mg}$ naproxen when co-administered with $60 \mathrm{mg}$ atogepant to fasted healthy participants on a semi-logarithmic scale

\subsection{Safety and Tolerability}

Overall incidences of treatment-emergent AEs were low; proportions of participants with treatment-emergent AEs considered related to study intervention ranged from 2.8 to $13.2 \%$ across the five study interventions. No SAEs, fatal events, TEAEs leading to discontinuation, or potential Hy's law cases were reported. The higher rate of TEAEs with atogepant alone or co-administered with acetaminophen was not attributable to any particular type or pattern of TEAEs. Most events were mild in intensity except for one event of moderate dysmenorrhea after dosing with $60 \mathrm{mg}$ atogepant and one event of moderate oropharyngeal pain after dosing with $1000 \mathrm{mg}$ acetaminophen. The most commonly reported TEAEs were oropharyngeal pain $(n=2$; after dosing with atogepant and considered to be not related to intervention) and nausea $(n=2$; after dosing with atogepant co-administered with acetaminophen and considered to be related to intervention).

Five participants had potentially clinically significant abnormal laboratory values. At the end of dosing, one participant had: hematocrit ratio less than 0.9 times the lower limit of normal (LLN), hemoglobin less than 0.9 times LLN, and red blood cell count less than 0.9 times LLN; one had absolute neutrophil count less than 0.7 times LLN; one had white blood cell count less than 0.9 times LLN; and two 
Table 3 Mean (SD) pharmacokinetic parameters of acetaminophen or naproxen when administered alone or when co-administered with atogepant

\begin{tabular}{|c|c|c|c|c|}
\hline \multirow[b]{2}{*}{ Pharmacokinetic parameters } & \multicolumn{2}{|c|}{ Acetaminophen pharmacokinetics } & \multicolumn{2}{|l|}{ Naproxen pharmacokinetics } \\
\hline & $\begin{array}{l}\text { Acetaminophen } \\
1000 \mathrm{mg}(n=39)\end{array}$ & $\begin{array}{l}\text { Acetaminophen } 1000 \mathrm{mg}+ \\
\text { atogepant } 60 \mathrm{mg}(n=38)\end{array}$ & Naproxen $500 \mathrm{mg}(n=36)$ & $\begin{array}{l}\text { Naproxen } 500 \mathrm{mg} \\
+ \text { atogepant } 60 \mathrm{mg} \\
(n=38)\end{array}$ \\
\hline$C_{\max }(\mu \mathrm{g} / \mathrm{mL})$ & $15.82(5.25)$ & $15.04(6.54)$ & $79.86(12.27)$ & $74.52(14.71)$ \\
\hline $\mathrm{AUC}_{0-t}(\mu \mathrm{g} \cdot \mathrm{h} / \mathrm{mL})$ & $60.80(18.61)$ & $59.32(18.42)$ & $1210.00(170.55)$ & $1183.01(186.88)$ \\
\hline $\mathrm{AUC}_{0-\infty}(\mu \mathrm{g} \cdot \mathrm{h} / \mathrm{mL})$ & $62.30(19.01)$ & $60.97(18.86)$ & $1292.47(201.56)$ & $1268.05(218.17)$ \\
\hline$T_{\max }(\mathrm{h})^{\mathrm{a}}$ & $1.00(0.50-2.00)$ & $1.50(0.50-4.00)$ & $1.00(0.50-4.00)$ & $1.50(0.50-6.00)$ \\
\hline$t_{1 / 2}(\mathrm{~h})$ & $4.67(1.48)$ & $5.16(1.68)$ & $18.66(3.00)$ & $19.14(2.61)$ \\
\hline$V_{\mathrm{z}} / F(\mathrm{~L})$ & $117.16(46.91)$ & $132.39(53.52)$ & $10.55(1.77)$ & $11.05(1.55)$ \\
\hline $\mathrm{CL} / F(\mathrm{~L} / \mathrm{h})$ & $17.80(6.29)$ & $18.54(8.26)$ & $0.40(0.06)$ & $0.41(0.07)$ \\
\hline
\end{tabular}

$A U C_{0-t}$ area under the plasma drug concentration-time curve (AUC) from time 0 to time $t, A U C_{0-\infty}$ AUC from time 0 to infinity, $C L / F$ apparent total body clearance of drug from plasma after extravascular administration, $C_{\max }$ maximum plasma drug concentration, $t_{1 / 2}$ terminal elimination half-life, $T_{\max }$ time to $C_{\max }, V_{z} / F$ apparent volume of distribution during the terminal phase after extravascular administration

${ }^{a}$ Median (range)

Table 4 Statistical analysis of pharmacokinetic parameters of plasma atogepant and acetaminophen administered alone and when co-administered

\begin{tabular}{|c|c|c|c|c|c|c|}
\hline & \multirow[t]{2}{*}{ Pharmacokinetic parameters } & \multicolumn{2}{|c|}{ Geometric LSM } & \multirow{2}{*}{$\begin{array}{l}\text { GMR (test/ } \\
\text { reference) }\end{array}$} & \multirow[t]{2}{*}{$90 \%$ lower CI } & \multirow[t]{2}{*}{$90 \%$ upper $\mathrm{Cl}$} \\
\hline & & Test & Reference & & & \\
\hline \multirow{3}{*}{$\begin{array}{l}\text { Plasma atogepant } \\
\text { Test }=\text { Atogepant }+ \\
\text { Acetaminophen Refer }- \\
\text { ence }=\text { Atogepant }\end{array}$} & $C_{\max }(\mathrm{ng} / \mathrm{mL})$ & 717.17 & 717.19 & 1.00 & 0.90 & 1.11 \\
\hline & $\mathrm{AUC}_{0-t}(\mathrm{ng} \cdot \mathrm{h} / \mathrm{mL})$ & 3794.44 & 3349.76 & 1.13 & 1.05 & 1.22 \\
\hline & $\mathrm{AUC}_{0-\infty}(\mathrm{ng} \cdot \mathrm{h} / \mathrm{mL})$ & 3828.79 & 3395.58 & 1.13 & 1.04 & 1.22 \\
\hline \multirow{3}{*}{$\begin{array}{l}\text { Plasma acetaminophen } \\
\text { Test }=\text { Atoge }- \\
\text { pant }+ \text { Acetaminophen } \\
\text { Reference }= \\
\text { Acetaminophen }\end{array}$} & $C_{\max }(\mu \mathrm{g} / \mathrm{mL})$ & 13.27 & 14.96 & 0.89 & 0.81 & 0.97 \\
\hline & $\mathrm{AUC}_{0-t}(\mu \mathrm{g} \cdot \mathrm{h} / \mathrm{mL})$ & 54.13 & 57.88 & 0.94 & 0.89 & 0.99 \\
\hline & $\operatorname{AUC}_{0-\infty}(\mu \mathrm{g} \cdot \mathrm{h} / \mathrm{mL})$ & 55.61 & 59.29 & 0.94 & 0.89 & 0.99 \\
\hline
\end{tabular}

$A U C_{0-t}$ area under the plasma drug concentration-time curve (AUC) from time 0 to time $t, A U C_{0-\infty}$ AUC from time 0 to infinity, $C_{m a x}$ maximum plasma drug concentration, GMR geometric means ratio, $L S M$ least squares mean

participants had potassium values greater than 1.1 times the upper limit of normal (ULN) at follow-up. Three participants had potassium values greater than 1.1 times ULN at interim assessments. None of the abnormal clinical laboratory results were reported as a TEAE, and none were considered to be clinically relevant.

Three participants had potentially clinically significant abnormal vital signs at the end of dosing, including one participant with diastolic $\mathrm{BP} \leq 50 \mathrm{mmHg}$ and decrease of $\geq 15 \mathrm{mmHg}$, one participant with pulse rate $\leq 50 \mathrm{bpm}$ and decreased $\geq 15 \mathrm{bpm}$, and one participant with body temperature $<35^{\circ} \mathrm{C}$. Four participants had abnormal vital signs during interim assessments, including one participant with diastolic $\mathrm{BP} \leq 50 \mathrm{mmHg}$ and decrease of $\geq 15 \mathrm{mmHg}$ (2 events); two participants with pulse rate of $\leq 50 \mathrm{bpm}$ and decrease of $\geq 15 \mathrm{bpm}$; and one participant with pulse rate $\geq 120 \mathrm{bpm}$ and increase of $\geq 15 \mathrm{bpm}$. None of the abnormal vital signs were reported as a TEAE, and none were considered to be clinically relevant. No potentially clinically significant abnormal ECG parameter values were reported.

\section{Discussion}

This drug-drug interaction study demonstrated that coadministration of $60 \mathrm{mg}$ atogepant with $1000 \mathrm{mg}$ acetaminophen or with $500 \mathrm{mg}$ naproxen resulted in no statistically or clinically relevant changes in $C_{\max }$ or overall systemic exposure to either drug and was safe and well tolerated. Similar to prior pharmacokinetic studies [26], atogepant was rapidly absorbed, with a $t_{\max }$ of $\sim 1.5 \mathrm{~h}$. Pharmacokinetic results following the co-administration of atogepant 


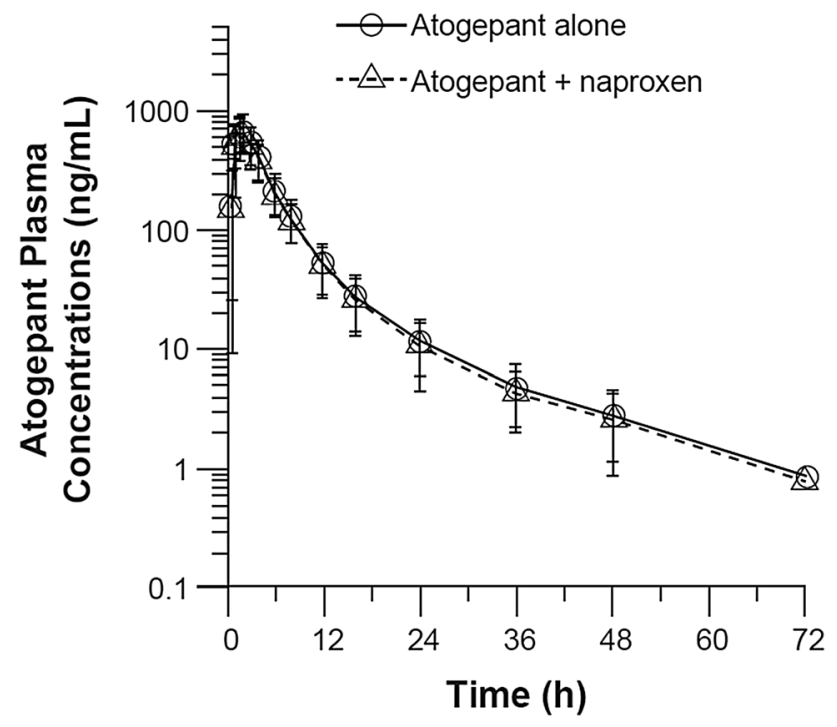

Fig. 4 Mean (SD) plasma atogepant concentration-time profile following single-dose oral administration of $60 \mathrm{mg}$ atogepant alone or when co-administered with $500 \mathrm{mg}$ naproxen to fasted healthy participants on a semi-logarithmic scale

with acetaminophen demonstrated an increase in the overall exposure (AUC) of atogepant by $13 \%$ and a decrease of acetaminophen $C_{\max }$ and AUC by $11 \%$ and $6 \%$, respectively. These changes are not expected to result in any clinically relevant effects on efficacy or safety. Based on the GMRs and $90 \%$ CIs, there was no drug-drug interaction following the co-administration of atogepant with naproxen. In a clinical trial to evaluate the risk of cardiac repolarization, atogepant administered at supratherapeutic doses of $300 \mathrm{mg}$ was safe and well tolerated in healthy adult participants. The small increase in atogepant AUC when co-administered with acetaminophen is therefore not likely to have an impact on the safety of atogepant.
Our observations of the lack of pharmacokinetic interactions between atogepant and acetaminophen or naproxen are consistent with our current understanding of how these medications are absorbed and metabolized. Atogepant, acetaminophen, and naproxen are all extensively metabolized in the liver, but through different mechanisms. Atogepant is a substrate of P-glycoprotein (P-gp) and the cytochrome P450 isoform CYP3A4 [26]. Acetaminophen is primarily metabolized by hepatic glucuronidation and sulfation [27], and naproxen is metabolized by CYP2C9 and CYP1A2 [28]. Despite the low likelihood of drug-drug interactions based on metabolic pathways, atogepant is likely to be co-administered with acetaminophen or naproxen for the acute treatment of migraine attacks, and this study is therefore important to confirm the safety and tolerability of these combinations.

Co-administration of atogepant with acetaminophen or naproxen was safe and well tolerated with no SAEs, deaths, or TEAEs leading to discontinuation. Treatment-emergent AEs were infrequent and mostly mild in severity. Although a slightly higher proportion of participants experienced TEAEs following co-administration of atogepant with acetaminophen compared with the other interventions, this increase was not attributable to any TEAE type or pattern.

Single-dose administration of atogepant was a limitation of the study. As a preventive medication for migraine, atogepant will be administered daily, and although atogepant does not accumulate upon repeated daily dosing, the pharmacokinetics of atogepant will be at steady state instead of being cleared between administrations as was done in this study. The interpretation of the results was limited by the small sample size, as is necessary for pharmacokinetic studies with intensive sampling, but the study was conducted according to guidance from the FDA [25], and the results were consistent with other pharmacokinetic studies of atogepant. Finally, the study was conducted in healthy adult participants, and

Table 5 Statistical analysis of pharmacokinetic parameters of plasma atogepant and naproxen administered alone and when co-administered

\begin{tabular}{|c|c|c|c|c|c|c|}
\hline & \multirow[t]{2}{*}{ Pharmacokinetic parameters } & \multicolumn{2}{|c|}{ Geometric LSM } & \multirow{2}{*}{$\begin{array}{l}\text { GMR (test/ } \\
\text { reference) }\end{array}$} & \multirow[t]{2}{*}{$90 \%$ lower CI } & \multirow[t]{2}{*}{$90 \%$ upper CI } \\
\hline & & Test & Reference & & & \\
\hline \multirow{3}{*}{$\begin{array}{l}\text { Plasma atogepant } \\
\text { Test }=\text { Atogepant }+ \\
\text { Naproxen Refer- } \\
\text { ence }=\text { Atogepant }\end{array}$} & $C_{\max }(\mathrm{ng} / \mathrm{mL})$ & 718.00 & 717.19 & 1.00 & 0.91 & 1.11 \\
\hline & $\mathrm{AUC}_{0-t}(\mathrm{ng} \cdot \mathrm{h} / \mathrm{mL})$ & 3312.38 & 3349.76 & 0.99 & 0.92 & 1.07 \\
\hline & $\mathrm{AUC}_{0-\infty}(\mathrm{ng} \cdot \mathrm{h} / \mathrm{mL})$ & 3351.17 & 3395.58 & 0.99 & 0.92 & 1.06 \\
\hline \multirow{3}{*}{$\begin{array}{l}\text { Plasma naproxen } \\
\text { Test }=\text { Atogepant }+ \\
\text { Naproxen Refer- } \\
\text { ence }=\text { Naproxen }\end{array}$} & $C_{\max }(\mu \mathrm{g} / \mathrm{mL})$ & 73.76 & 78.67 & 0.94 & 0.90 & 0.97 \\
\hline & $\mathrm{AUC}_{0-t}(\mu \mathrm{g} \cdot \mathrm{h} / \mathrm{mL})$ & 1172.17 & 1197.13 & 0.98 & 0.96 & 1.00 \\
\hline & $\mathrm{AUC}_{0-\infty}(\mu \mathrm{g} \cdot \mathrm{h} / \mathrm{mL})$ & 1253.82 & 1275.90 & 0.98 & 0.96 & 1.00 \\
\hline
\end{tabular}

$A U C_{0-t}$ area under the plasma drug concentration-time curve (AUC) from time 0 to time $t, A U C_{0-\infty}$ AUC from time 0 to infinity, $C_{\text {max }}$ maximum plasma drug concentration, GMR geometric means ratio, $L S M$ least squares mean 
the results may not be generalizable to individuals with migraine.

\section{Conclusions}

No drug-drug interactions were observed following coadministration of $60 \mathrm{mg}$ atogepant with either $1000 \mathrm{mg}$ acetaminophen or $500 \mathrm{mg}$ naproxen. Co-administration of atogepant with acetaminophen or naproxen was safe and well tolerated in the population of healthy adult participants. These results support the continued development of atogepant for migraine prevention.

Supplementary Information The online version contains supplementary material available at https://doi.org/10.1007/s40261-021-01034-5.

Acknowledgements The authors thank Abhijeet Jakate for conducting pharmacokinetic analyses. Writing and editorial assistance was provided to the authors by Peloton Advantage, LLC, an OPEN Health company, Parsippany, NJ, USA, and was funded by AbbVie.

\section{Declarations}

Funding This study was sponsored by Allergan (prior to its acquisition by AbbVie).

Conflicts of interest Ramesh Boinpally, Kayla Chen, and Matthew Butler are employees of AbbVie, and may hold AbbVie stock. John Spaventa was a contingent employee of AbbVie at the time of the study.

Ethics approval This study was conducted in accordance with the principles of the Declaration of Helsinki and the International Council for Harmonisation (ICH) E6 guideline for Good Clinical Practice. The study protocol was approved by Bio-Kinetic Clinical Applications IRB (Springfield, MO, USA).

Consent to participate All participants provided written informed consent prior to initiation of any study-specific procedures.

\section{Consent for publication Not applicable.}

Availability of data and material AbbVie is committed to responsible data sharing regarding the clinical trials we sponsor. This includes access to anonymized, individual and trial-level data (analysis data sets), as well as other information (e.g., protocols and Clinical Study Reports), as long as the trials are not part of an ongoing or planned regulatory submission. This includes requests for clinical trial data for unlicensed products and indications. This clinical trial data can be requested by any qualified researchers who engage in rigorous, independent scientific research, and will be provided following review and approval of a research proposal and Statistical Analysis Plan (SAP) and execution of a Data Sharing Agreement (DSA). Data requests can be submitted at any time and the data will be accessible for 12 months, with possible extensions considered. For more information on the process, or to submit a request, visit the following link: https://www. abbvie.com/our-science/clinical-trials/clinical-trials-data-and-infor mation-sharing/data-and-information-sharing-with-qualified-resea rchers.html.
Code availability Not applicable.

Author contributions Study design: JS; RB. Collection and assembly of data: JS; RB; KC. Data analysis: RB; KC. Data interpretation: all authors. Manuscript review and revisions: All authors. Final approval of manuscript: All authors

Open Access This article is licensed under a Creative Commons Attribution-NonCommercial 4.0 International License, which permits any non-commercial use, sharing, adaptation, distribution and reproduction in any medium or format, as long as you give appropriate credit to the original author(s) and the source, provide a link to the Creative Commons licence, and indicate if changes were made. The images or other third party material in this article are included in the article's Creative Commons licence, unless indicated otherwise in a credit line to the material. If material is not included in the article's Creative Commons licence and your intended use is not permitted by statutory regulation or exceeds the permitted use, you will need to obtain permission directly from the copyright holder. To view a copy of this licence, visit http://creativecommons.org/licenses/by-nc/4.0/.

\section{References}

1. Headache Classification Committee of the International Headache Society. The International Classification of Headache Disorders, 3rd edition (beta version). Cephalalgia. 2013;33(9):629-808.

2. Pietrobon D, Moskowitz MA. Pathophysiology of migraine. Annu Rev Physiol. 2013;75:365-91.

3. World Health Organization. Headache disorders. 2016. http:// www.who.int/en/news-room/fact-sheets/detail/headache-disor ders. Accessed 21 Apr 2021.

4. Lipton RB, Bigal ME, Diamond M, Freitag F, Reed ML, Stewart WF. Migraine prevalence, disease burden, and the need for preventive therapy. Neurology. 2007;68(5):343-9.

5. Headache Classification Committee of the International Headache Society. The international classification of headache disorders, 3rd edition. Cephalalgia. 2018;38(1):1-211.

6. Global, regional, and national incidence, prevalence, and years lived with disability for 354 diseases and injuries for 195 countries and territories, 1990-2017: a systematic analysis for the Global Burden of Disease Study 2017. Lancet. 2018;392(10159):1789-858.

7. Global, regional, and national burden of migraine and tension-type headache, 1990-2016: a systematic analysis for the Global Burden of Disease Study 2016. Lancet Neurol. 2018;17(11):954-76.

8. Lipton RB, Manack Adams A, Buse DC, Fanning KM, Reed ML. A comparison of the chronic migraine epidemiology and outcomes (CaMEO) study and American Migraine Prevalence and Prevention (AMPP) Study: demographics and headache-related disability. Headache. 2016;56(8):1280-9.

9. American Headache Society. The American Headache Society position statement on integrating new migraine treatments into clinical practice. Headache. 2019;59(1):1-18.

10. Lew C, Punnapuzha S. Migraine medications. Treasure Island: StatPearls Publishing LLC; 2020.

11. Steiner TJ, Paemeleire K, Jensen R, Valade D, Savi L, Lainez $\mathrm{MJ}$, et al. European principles of management of common headache disorders in primary care. J Headache Pain. 2007;8(Suppl 1):S3-47.

12. Lanteri-Minet M, Valade D, Geraud G, Lucas C, Donnet A. Revised French guidelines for the diagnosis and management of migraine in adults and children. J Headache Pain. 2014;8(15):2. 
13. Diener H, Holle-Lee D, Nägel S, Dresler T, Gaul C, Göbel H, et al. Treatment of migraine attacks and prevention of migraine: Guidelines by the German Migraine and Headache Society and the German Society of Neurology. Clin Transl Neurosci. 2019;3(1):2514183X1882337.

14. Becker WJ. Acute migraine treatment in adults. Headache. 2015;55(6):778-93.

15. Bigal ME, Borucho S, Serrano D, Lipton RB. The acute treatment of episodic and chronic migraine in the USA. Cephalalgia. 2009;29(8):891-7.

16. Ubrelvy [package insert]. Madison: Allergan USA, Inc.; 2020.

17. Nurtec ODT [package insert]. New Haven: Biohaven Pharmaceuticals, Inc.; 2020.

18. Ajovy [package insert]. North Wales: Teva Pharmaceuticals USA, Inc.; 2020

19. Emgality [package insert]. Indianapolis: Eli Lilly and Company; 2019.

20. Aimovig [package insert]. Thousand Oaks: Amgen Inc., and Novartis Pharmaceuticals Corporation; 2020.

21. Vyepti [package insert]. Bothell: Lundbeck Seattle BioPharmaceuticals, Inc; 2020.

22. Charles A, Pozo-Rosich P. Targeting calcitonin generelated peptide: a new era in migraine therapy. Lancet. 2019;394(10210):1765-74.

23. Goadsby PJ, Dodick DW, Ailani J, Trugman JM, Finnegan M, Lu K, et al. Safety, tolerability, and efficacy of orally administered atogepant for the prevention of episodic migraine in adults: a double-blind, randomised phase $2 \mathrm{~b} / 3$ trial. Lancet Neurol. 2020;19(9):727-37.

24. Ailani J, Lipton RB, Goadsby PJ, Guo H, Miceli R, Severt L, et al. Atogepant significantly reduces mean monthly migraine days in the phase 3 trial (ADVANCE) for the prevention of migraine [abstract MTV20-OR-018]. Cephalalgia. 2020;40(1 suppl):15-6.

25. Clinical Drug Interaction Studies-Study Design, Data Analysis, and Clinical Implications Guidance for Industry. 2017 October 24, 2017. https://www.fda.gov/media/82734/download

26. Ankrom W, Xu J, Vallee MH, Dockendorf MF, Armas D, Boinpally R, et al. Atogepant has no clinically relevant effects on the pharmacokinetics of an ethinyl estradiol/levonorgestrel oral contraceptive in healthy female participants. J Clin Pharmacol. 2020;60(9):1157-65.

27. Jozwiak-Bebenista M, Nowak JZ. Paracetamol: mechanism of action, applications and safety concern. Acta Pol Pharm. 2014;71(1):11-23.

28. Miners JO, Coulter S, Tukey RH, Veronese ME, Birkett DJ. Cytochromes P450, 1A2, and 2C9 are responsible for the human hepatic O-demethylation of R- and S-naproxen. Biochem Pharmacol. 1996;51(8):1003-8. 